\title{
Monochromated Electron Energy-Loss Spectroscopy of Lead-Free Halide Perovskite Semiconductors
}

\author{
Jessica A. Alexander ${ }^{1}$, David W. McComb ${ }^{1}$, Eric T. McClure ${ }^{2}$ and Patrick M. Woodward ${ }^{2}$ \\ 1. Center for Electron Microscopy and Analysis, Department of Materials Science and Engineering, The \\ Ohio State University, Columbus, $\mathrm{OH}$, United States \\ 2. Department of Chemistry and Biochemistry, The Ohio State University, Columbus, OH, United States
}

Over the last few years, the performance of perovskite solar cells has improved considerably with efficiencies greater than $22 \%$ reported [1]. While this rapid rise in efficiency make them an extremely promising alternative to traditional silicon solar cells, perovskite solar cells suffer from stability issues in light and air. There are also ecological and toxicity concerns as the perovskites used in these devices contain lead. To address the latter toxicity concern, lead-free halide perovskite semiconductors have been developed recently [2]. These lead-free halide perovskites have similar band gaps and reflectance behavior as their lead-containing counterparts and could, ultimately, provide a safer alternative to the perovskite materials currently utilized in solar cell devices [2]. Knowledge of their optoelectronic properties is critical to their further development and utilization in actual solar cell devices.

Electron energy-loss spectroscopy (EELS) is a powerful tool form measuring optoelectronic properties of materials. Utilizing a monochromated electron beam in a scanning transmission electron microscope (STEM), it is possible to collect EELS spectra with high spatial and high energy resolutions, over a large energy range encompassing ultraviolet, visible, and infrared wavelengths (and beyond). From these EELS spectra, optoelectronic properties such as the real $\left(\varepsilon_{1}\right)$ and imaginary $\left(\varepsilon_{2}\right)$ parts of the complex dielectric function, the refractive index $(n)$ and the extinction coefficient $(\kappa)$, and the optical absorption coefficient $(\alpha)$ can be extracted [3].

To probe the optoelectronic properties of these lead-free halide perovskites, EELS measurements were made using a monochromated $60 \mathrm{kV}$ electron beam in a FEI Titan ${ }^{3}$ 60-300 aberration-corrected S/TEM. In Figure 1, the raw EELS spectrum is shown for one of these materials, $\mathrm{Cs}_{2} \mathrm{NaBiCl}_{6}$. In the spectrum, it is possible to identify both the bismuth $\mathrm{O}_{4,5}$ edge (at $\sim 27 \mathrm{eV}$ ) and the sodium $\mathrm{L}_{2,3}$ edge (at $\sim 33 \mathrm{eV}$ ). There are also multiple peaks detected at energy-losses below $10 \mathrm{eV}$, some of which may be associated with single electron transitions in the material. Examination of the $\varepsilon_{1}$ and $\varepsilon_{2}$ spectra obtained from the EELS data via Kramers-Kronig analysis can be used to distinguish between single electron transitions and collective excitations (Figure 2) [3]. Peaks in $\varepsilon_{2}$ correspond to single electron transitions, and examination of the $\varepsilon_{2}$ spectrum (Figure 2) suggests that there are three intense single electron transitions in this material below $10 \mathrm{eV}$. These energy values can be compared with its band energy diagram to identify these transitions. Since EELS can be collected over a large energy range with high energy and high spatial resolution, once actual devices are made comprised of these lead-free halide perovskites, it will be possible to track changes in the local chemistry and bonding (from $\varepsilon_{2}$ ) across interfaces of the device. Data has been shown here for only one of these lead-free halide perovskites. Results and analysis from this and the other lead-free halide perovskites will be discussed as a part of this contributed work [4]. 


\section{References:}

[1] http://www.nrel.gov/pv/assets/images/efficiency-chart.png

[2] E.T. McClure et al, Chem. Mater. 28 (2016), p. 1348.

[3] R.F. Egerton in "Electron Energy-Loss Spectroscopy in the Electron Microscope", (Springer, New York).

[4] The authors would like to thank all collaborators and technical support at both the Center for Electron Microscopy (CEMAS) at The Ohio State University and the Materials and Manufacturing Directorate at the Air Force Research Laboratory (AFRL) at Wright-Patterson Air Force Base. Funding was provided by an AFRL/DAGSI Ohio-Student Faculty Research Fellowship awarded by the Air Force Laboratory Manufacturing and Materials Directorate, and by The Ohio State University through a Distinguished University Fellowship.
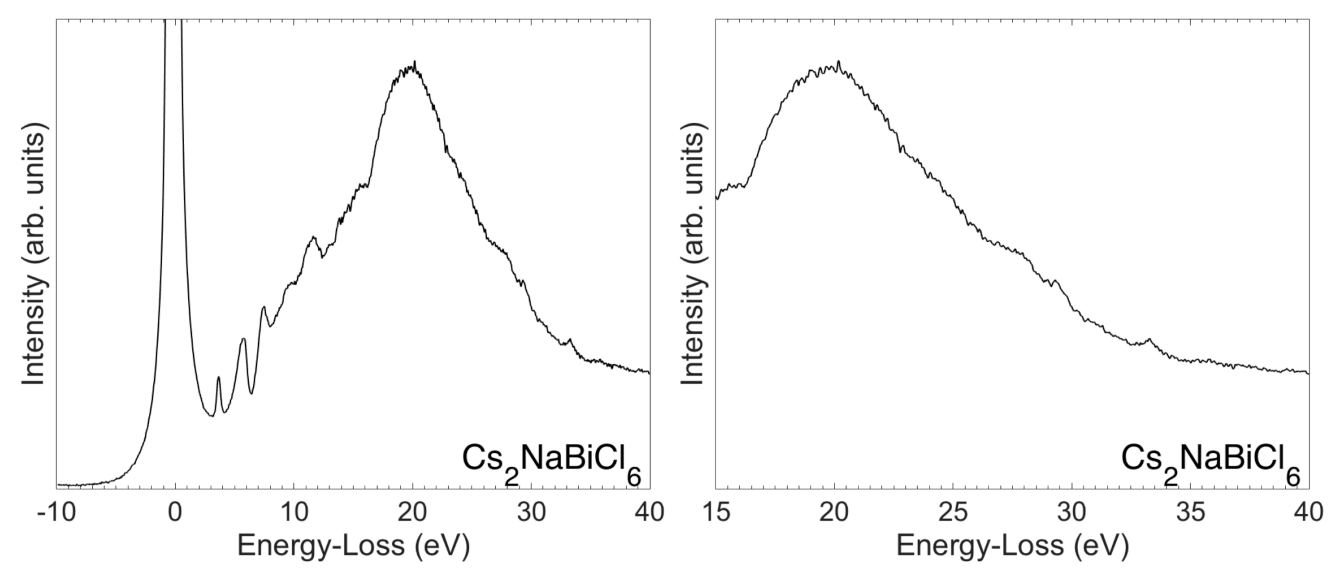

Figure 1. (a) Raw EELS spectrum collected for $\mathrm{Cs}_{2} \mathrm{NaBiCl}_{6}$ and (b) zoomed in on the bismuth and sodium edges.
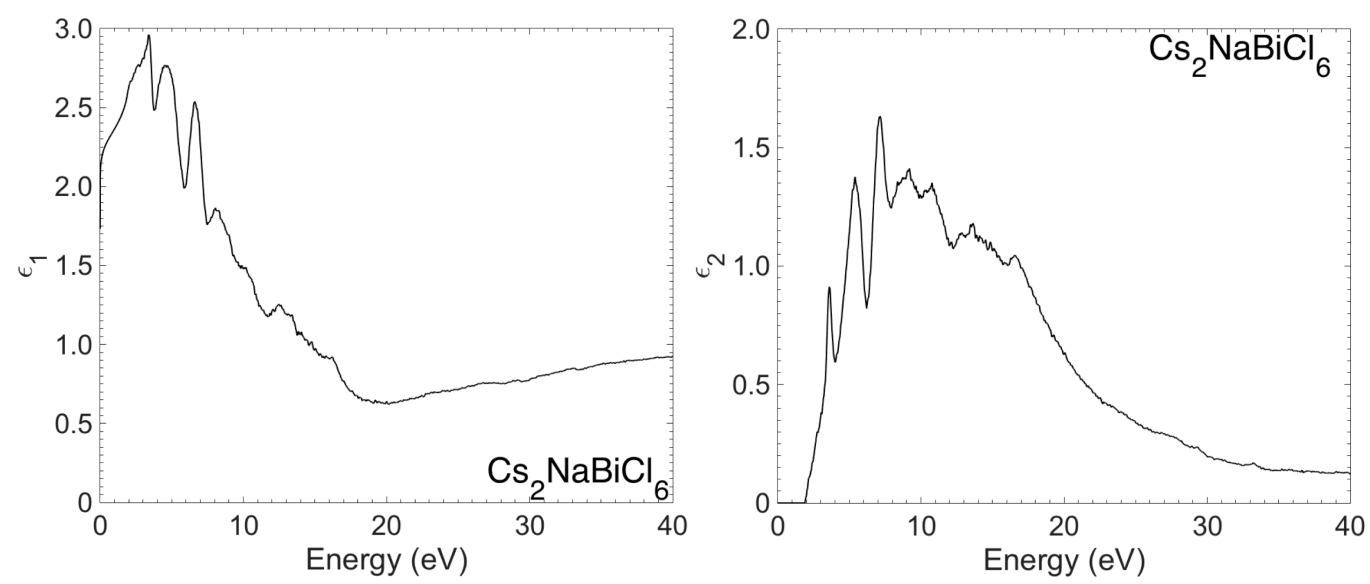

Figure 2. The real (left) and imaginary (right) parts of the complex dielectric function of $\mathrm{Cs}_{2} \mathrm{NaBiCl}_{6}$ that was determined via Kramers-Kronig analysis of the EELS spectrum. 